\title{
Fronteras intraeuropeas, desactivación fronteriza, cooperación transfronteriza e instituciones: El caso de La Raya ibérica
}

\section{Borders within Europe, border deactivation, cross-border cooperation and institutions: The Iberian Raya case}

Francisco José Calderón Vázquez a* (i) (http://orcid.org/0000-0001-8168-2549)
Recibido el 2 de agosto de 2016.

Aceptado el 27 de enero de 2017.

*Autor para correspondencia: Francisco José Calderón Vázquez, correo electrónico: fjcalderon@ uma.es

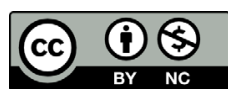

Todos los contenidos de Estudios Fronterizos se publican bajo la licencia Creative Commons Atribución no comercial 2.5 México, y pueden ser usados gratuitamente para fines no comerciales, dando el crédito a los autores y a la revista Estudios Fronterizos.
${ }^{\text {a } U n i v e r s i d a d ~ d e ~ M a ́ l a g a, ~ F a c u l t a d ~ d e ~ C i e n c i a s ~ E c o n o ́ m i c a s, ~ D e p a r t a m e n t o ~ d e ~ E c o n o m i ́ a ~ A p l i c a d a, ~}$ Málaga, España, correo electrónico: fjcalderon@uma.es

\section{Resumen}

El presente trabajo plantea una aplicación al caso de la política europea de cooperación transfronteriza (CTF). Se revisa la CTF llevada a cabo en la frontera hispano-portuguesa desde una perspectiva funcional, mediante una descripción panorámica del proceso de implementación de CTF luso-hispana, de los agentes e instituciones que la han llevado a cabo, en particular las comunidades de trabajo, y de los resultados logrados a lo largo del proceso. Entendiendo por resultados: 1) qué avances o retrocesos se han realizado en la desactivación de esta frontera secular —es decir, se ha logrado eliminar el efecto frontera-, 2) qué obstáculos quedan pendientes y 3) cuáles son los desafíos de futuro. Las conclusiones muestran un panorama ambiguo por cuanto que se constatan grandes avances en los aspectos de infraestructura, de transporte, comunicación, intercambio y comercio; aunque todavía queden flecos pendientes y otros campos — culturales, antropológicos-sociológicos-donde los progresos son poco significativos o nulos.

Palabras clave: fronteras interiores europeas, desactivación fronteriza, cooperación transfronteriza, instituciones.

\section{Abstract}

The present paper presents an application to the case of the European cross-border cooperation policy ( $\mathrm{CBC})$. Specifically, the $\mathrm{CBC}$ carried out at the Spanish-Portuguese border is reviewed from a functional perspective. To do this, a descriptive overview of the implementation process of Portuguese-Spanish CBC is made, showing the agents and institutions that have carried it out, in particular the working communities, and the results achieved throughout the process. Understanding by results: 1) what progress or recoils have been made in

CÓMO CITAR: Calderón, F. J. (2017). Fronteras intraeuropeas, desactivación fronteriza, cooperación transfronteriza e instituciones: El caso de La Raya ibérica [Borders within Europe, border deactivation, cross-border cooperation and institutions: The Iberian Raya case]. Estudios Fronterizos, 18(36), 78-101, doi: 10.21670/ref.2017.36.a04 
the deactivation of this secular border - that is, the border effect has been eliminated-, 2) what obstacles remain outstanding and 3) what are the future challenges.

Keywords: EU internal borders, border deactivation, cross border cooperation, institutions.

\section{Introducción: Integración europea y enfoque transfronterizo}

Desde una perspectiva europea, la generación del Mercado Único Europeo (muE) constituía el eje central de la Comunidad Económica Europea en su momento y de la actual Unión Europea (UE). Dicho mercado común y la subsiguiente integración económica europea, se consideraban como instrumentos de gran eficacia de cara a la consecución de un crecimiento económico estable y duradero junto a un incremento de los niveles de vida en el viejo continente. ${ }^{1}$ La puesta en marcha del MuE implicaba lograr altos niveles de integración económica entre los diferentes estados miembros de la UE. Lo que hacía necesario eliminar las diferentes barreras y obstáculos que impedían la integración. De ahí que resultara imprescindible desactivar las fronteras intracomunitarias, lo que explica que la remoción de las mismas haya constituido uno de los cursos fundamentales de acción en el proceso constitutivo de la uE.

Lo anterior no debería resultar extraño puesto que en Europa desde la Paz de Westfalia (1648), ${ }^{2}$ el Tratado de los Pirineos $(1659)^{3}$ y los Tratados de Utrecht-Rastatt (1713-1715 $)^{4}$ el territorio englobado dentro de las fronteras de un determinado estado constituye su espacio de soberanía, sometido al ordenamiento jurídico-político del estado en cuestión. Por ello, la existencia de una frontera política implica la discontinuidad o diferencial jurídico, político, económico, fiscal, aduanero, lingüístico-étnico-cultural, etcétera, entre territorios limítrofes (Calderón, 2015, p. 66), al pertenecer estos a diferentes estados, cada uno con su propio sistema normativo, político y económico-fiscal; sistemas que terminan y comienzan en la frontera (Cairo y Lois, 2013; Foucher, 2007). Por ello, "un mundo de estados soberanos es un mundo dividido por las líneas fronterizas" (Taylor, 1994, p. 152).

${ }^{1}$ No en vano, en la Versión Consolidada del Tratado Constitutivo de la Comunidad Europea (2002, art. 2, p. 40):

La Comunidad tendá por misión promover, mediante el establecimiento de un mercado común y de una unión económica y monetaria y mediante la realización de las políticas o acciones comunes contempladas en los artículos 3 y 4 , un desarrollo armonioso, equilibrado y sostenible de las actividades económicas en el conjunto de la Comunidad, un alto nivel de empleo y de protección social, la igualdad entre el hombre y la mujer, un crecimiento sostenible y no inflacionista, un alto grado de competitividad y de convergencia de los resultados económicos, un alto nivel de protección y de mejora de la calidad del medio ambiente, la elevación del nivel y de la calidad de vida, la cohesión económica y social y la solidaridad entre los Estados miembros.

${ }^{2}$ La Paz de Westfalia inició un nuevo orden en Europa basado en los conceptos de soberanía nacional e integridad territorial, fundamento de la existencia de los Estados-Nación, frente a la concepción feudal de que territorios y pueblos constituían un patrimonio hereditario.

${ }^{3}$ Donde se define con precisión la frontera entre los reinos de Francia y España siguiendo la línea de los Pirineos.

${ }^{4}$ El Tratado de Utrecht-Rastatt pone fin a la Guerra de Sucesión Española, generándose una serie de nuevos estados que se reconocen por sus fronteras. 
La discontinuidad provoca altos costes de transacción que pueden derivar tanto de la exigibilidad de acuerdos y contratos más allá del confín, como de los diferenciales lingüísticos y culturales existentes, de las incertidumbres en el tipo de cambio, de la pertenencia a diferentes sistemas monetarios, etcétera. Por ello, la discontinuidad fronteriza genera el denominado, efecto frontera (Chen, 2004; Evans, 2003; Gil-Pareja, Llorca-Vivero, Martínez-Serrano y Oliver, 2005; Minondo, 2007; Nitsch, 2000), que dificulta y obstaculiza los intercambios fronterizos (Rodrik, 2012).

A costes de transacción y efecto frontera se les ha de sumar el denominado efecto callejón sin salida (Baigorri y Cortés, 1997; Calderón, 2010), al clausurar la frontera el territorio estatal, las redes de infraestructuras y comunicaciones de dicho estado (carreteras, caminos, vías férreas y redes energéticas) mueren en la línea fronteriza. Solo a través de los pasos fronterizos internacionales abiertos (discrecionalmente) para el efecto, tienen continuidad las comunicaciones. Por ello, surgen situaciones de desarticulación territorial tanto con el resto del estado de pertenencia como con los territorios del otro lado de la frontera, al estar desvinculadas o pobremente comunicadas con ambos.

En el contexto de la construcción del mue, la problemática fronteriza (y sus corolarios: costes de transacción, efectos frontera y callejón sin salida), van a ser abordados desde un planteamiento interactivo que considera a las fronteras intracomunitarias como tales "espacios transfronterizos" (Dörrenbacher y Brücher, 2000; Gildersleeve, 1976) es decir: zonas de contacto, conexión y cooperación entre territorios y comunidades contiguos (Anderson, O'Dowd y Wilson, 2003; Wackermann, 2003). Ello suponía un cambio cualitativo espectacular en el tratamiento y gestión de las zonas fronterizas intereuropeas, hasta ese momento consideradas meros bordes exteriores del Estado. Al contactar cotidianamente con los "otros" europeos, los territorios fronterizos disponían de un capital social singular que les permitía actuar como laboratorios propulsores del muE y, por ende, del proceso integrador (Amilhat-Szary y Fourny, 2006; Anderson et al. 2003; Dupeyron, 2008; Harguindéguy, 2007; Houtum y Strüver, 2002; Kramsch y Hooper, 2004; Perkmann y Sum, 2002).

Convertir las fronteras intracomunitarias en motores de la integración europea suponía un desafío de calibre histórico que superaba, con mucho, el voluntarismo europeísta. Nunca han sido fáciles las relaciones entre vecinos europeos, lo evidencia una historia cargada de conflictos que surgían, precisamente, en las marcas fronterizas. Asimismo, la propia complejidad de la desactivación fronteriza, al implicar un proceso multinivel $^{5}$ (y bilateral), convierte al proceso desfronterizador en actividad de difícil implementación e incierto resultado.

Para superar el abismo, la Comisión Europea propuso desarrollar la interacción entre zonas fronterizas empleando la cooperación transfronteriza (CTF) como instrumento básico, si bien desde una perspectiva multivariable (incluyendo aspectos institucionales, económicos, infraestructurales, ambientales, culturales, renta, nivel de vida, etcétera), incentivando el conocimiento y la comprensión de los "otros" (Asociación de las Regiones Fronterizas Europeas [ARFE], 1997). Paralelamente, se ofrecía desde

\footnotetext{
${ }^{5} \mathrm{Al}$ comprender diferentes dimensiones que van desde la eliminación de barreras físicas, hasta la reducción o minimización de los costes de transacción, pasando por la comprensión y aceptación del "otro".

${ }^{6}$ Lo que suponía conocer las particularidades culturales, lingüísticas y económicas de los territorios colindantes, fomentándose la cercanía y el conocimiento mutuo para superar perjuicios y hostilidades históricas.
} 
la Política Regional Europea todo un catálogo de incentivos económicos dirigidos a potenciar el desarrollo socioeconómico fronterizo ${ }^{7}$ (ARFE, 1997; Heredero y Olmedillas, 2009), estimulándose tanto a administraciones públicas (estatales, regionales y locales), como a la sociedad civil (ciudadanos, universidades, asociaciones, fundaciones, sindicatos, entre otros), al cambio de conducta respecto a las fronteras; estímulos sin los cuales, difícilmente, podría hablarse de la actual Europa sin fronteras.

¿Hasta qué punto estos designios europeístas se han llevado a cabo? ¿Hasta qué punto esa sugestiva estrategia basada en la cooperación transfronteriza ha dado sus frutos? La respuesta a cuestiones tan complejas excedería con mucho las posibilidades de este trabajo, al comprender un conjunto heterogéneo de situaciones entre diferentes fronteras intracomunitarias; pero sí que podemos analizar una aplicación de la política transfronteriza europea a un caso concreto: la frontera luso-hispana, la denominada Raya ibérica, secular frontera intraeuropea que, como un gran plano vertical, ha separado a los dos grandes Estados ibéricos durante más de 700 años (Podadera y Calderón, 2014).

Toda vez definido el sujeto de estudio, cabría preguntarse en términos genéricos ¿Cómo ha funcionado la cooperación transfronteriza en la frontera luso-hispana? ¿Qué instituciones la han llevado a cabo? y ya más concretamente, la profusión de iniciativas CTF observada en La Raya desde 1991 unida a la presencia de importantes estímulos económicos ¿Ha contribuido a eliminar o minimizar la suma de los efectos frontera y callejón sin salida? Y, en definitiva ¿Se han reducido sustantivamente los costes de transacción en la frontera hispano-lusa?

A estas cuestiones pretende dar respuesta el presente trabajo, donde revisamos una aplicación de la experiencia europea transfronteriza a un caso singular: La Raya ibérica, ${ }^{8}$ describiendo su proceso de implementación, las instituciones CTF que la han desarrollado (en particular las comunidades de trabajo) y sus resultados. El foco del análisis se centra en los progresos que se han venido realizando (o no) en la desactivación de esta frontera secular, qué obstáculos quedan pendientes y cuáles son los desafíos del futuro.

El presente texto se encuadraría en la modalidad de artículo de revisión, por ello orientado al análisis y sistematización de investigaciones publicadas sobre diferentes aspectos que confluyen en la temática de la cooperación transfronteriza y de sus aplicaciones al caso hispano-portugués, ${ }^{9}$ de ahí que los aspectos cuantitativos presenten un desarrollo meramente descriptivo.

A tal fin y tras esta introducción, el texto se ha estructurado en tres secciones más: En la primera, revisamos las relaciones institucionalidad/cooperación transfronteriza desde tres diferentes perspectivas: teórica, instrumental y empírico-europea, planteando

\footnotetext{
${ }^{7}$ Mediante los Fondos Estructurales (art. 10 del Fondo Europeo de Desarrollo Regional [FEDER]) y programas específicos como Interreg en sus sucesivas ediciones: Interreg: I (1989-1993); Interreg IIA+PHARE/ TACIS-CBC (1994-1999); Interreg III (2000-2006); Interreg IV (2007-2013) Interreg V-A (2014-2020).

${ }^{8}$ Para una profundización en la temática fronteriza hispano-lusa ver Calderón (2015), Medina (2007), Cairo, Godinho y Pereiro (2009), Macorra (2005) y López (2005).

${ }^{9}$ En la temática CTF, destacan las recientes aportaciones de Medeiros (2010; 2015), Lois y Carballo (2015), Domínguez y Varela (2015), Fernández (2008), Oliveras, Durà y Perkmann (2010). Por lo que respecta al impacto de la CTF es de resaltar la aportación de Farinós y Payà (2005).
} 
las coordenadas conceptuales y teóricas esenciales. En la segunda, procedemos a una panorámica evolutiva de la institucionalidad CTF hispano-lusa, centrada en la evolución de comunidades y grupos de trabajo, revisándose su origen, configuración y evolución, así como sus características organizativas y funcionales. En la sección tercera, revisamos de forma sintética los resultados de casi treinta años de CTF hispano-lusa, planteando su impacto sobre la problemática fronteriza (efecto frontera, callejón sin salida y costes de transacción), analizándose la situación de partida, cambios observados, avances, progresos y obstáculos remanentes. Por último, procedemos a las conclusiones y reflexiones.

\section{Institucionalidad y cooperación transfronteriza}

Si las instituciones son la respuesta a los dilemas que genera la acción colectiva (North, 1990; Olson, 1965), la gestión de los complicados procesos de desactivación fronteriza suponía contar con "instituciones" que por un lado, regulasen las conductas de los agentes transfronterizos, incentivando la cooperación y, por otro, dirigieran los procesos desfronterizadores dándole continuidad temporal a los mismos.

Podemos abordar las relaciones entre cooperación transfronteriza e instituciones desde tres perspectivas: instrumental, empírica-europea y teórica. Desde una perspectiva instrumental, la CTF aparece como instrumento para la superación de la problemática fronteriza, constituyendo su principal objetivo la supresión de los obstáculos distorsionadores provocados por el efecto frontera (ARFE, 1997). A fines de la Segunda Guerra Mundial, en la destrozada Europa, se hizo evidente la necesidad de dotar de mayor eficacia a las iniciativas de desarrollo orientadas a atender la problemática fronteriza. Para ello, estas debían ser concertadas (y en la medida de lo posible ejecutadas) bilateralmente por las administraciones públicas y restantes actores fronterizos. Con ello se promovía, por vez primera, un planteamiento de desarrollo que enfatizaba la intensificación de las relaciones transfronterizas. Tales interacciones podían ya preexistir en ciertos casos, presentando un carácter espontaneo (Julio, 2012) de mero intercambio entre ambas partes, pero en otros no. En estos la cooperación tenía que ser estimulada e incentivada.

Desde la perspectiva Empírico-Europea, la CTF (y las instituciones que la susten$\tan$ ), aparecen como una herramienta para la construcción de las denominadas "Europa Sin Fronteras" y "Europa de las Regiones", ideas cardinales que, con sus luces y sombras, han guiado la experiencia europea de integración (Scott y Collins, 1997). No en vano, según la ARFE, la razón última de la CTF radica en la superación de las negativas consecuencias del efecto frontera, para lo cual se propone la creación de espacios transfronterizos de servicios, la reducción de las fronteras intracomunitarias a simples demarcaciones administrativas y, por último, a largo plazo, la conversión de la posición periférica de las regiones fronterizas en una posición central dentro de la UE (ARFE, 1997, p. 6).

Si la construcción de la "Europa sin fronteras" suponía o implicaba una suerte de revolución territorial, sus bases sociales tenían que ser amplias. De ahí, el énfasis en la participación del conjunto de agentes fronterizos: desde gobiernos nacionales, regionales y locales, hasta la sociedad civil organizada, pasando por actores privados y organizaciones no gubernamentales. Como mejores conocedores de la problemática 
fronteriza en cuestión, dichos actores tenían que protagonizar su solución mediante la CTF. Por eso, en la experiencia europea la CTF presenta un carácter de encrucijada, de cruce de caminos, donde una profusión de sujetos interactúa, generándose mapas de relaciones.

Dado que la CTF resultaba esencial para lograr la Europa sin fronteras, esta tenía que ser incentivada, por lo que la ayuda financiera proveniente de la Comisión Europea resulta un factor clave en la creación de la institucionalidad transfronteriza (Boman, 2005; Perkmann, 2003). En estas coordenadas, las instituciones CTF aparecen cargadas de dualidad, puesto que, por un lado, son el resultado de los programas europeos de incentivos (al ser creadas ex profeso para desarrollarlos) y, del otro, son "hacedores" de la política europea transfronteriza, ya que al desarrollar los programas europeos CTF, la llevan a cabo.

En la experiencia europea, surge toda una serie de organizaciones destinadas a gestionar la CTF (con acceso efectivo a los recursos financieros Interreg), destacando tanto las denominadas comunidades de trabajo (que desarrollamos para el caso hispano-luso en el apartado siguiente), como a las más conocidas Euroregions, que son instituciones paradigmáticas del escenario transfronterizo UE. Posiblemente porque su nacimiento ${ }^{10}$ es previo al catálogo de incentivos y programas UE, personificando esa cooperación espontanea e histórica entre europeos, que aunque tenían distinta nacionalidad condividían una cultura común europea, visión un tanto romántica pero efectiva.

Comunidades de trabajo y euroregiones han institucionalizado la CTF, posibilitando su continuidad (Boira, 2004; Gabbe, 2004). Ello no significa que todo sean luces; vistos los resultados bastante dispares de los proyectos CTF, ${ }^{11}$ fruto de la heterogeneidad de tipologías fronterizas existentes en la UE y el consiguiente diferencial de intensidad de las interacciones fronterizas (Council of Europe, 2006; 2009).

No debería omitirse el enorme efecto demostración generado por la experiencia CTF europea, cuyo eco se ha expandido a otros continentes. Destacando las iniciativas CTF en fronteras latinoamericanas (Coletti, 2010; Rhi-Sausi y Conato, 2009).

Desde la perspectiva teórica, la CTF es uno de los campos esenciales de los denominados Estudios de Frontera, habiéndose abordado su estudio desde los enfoques de la elección racional y de la economía neoclásica, ${ }^{12}$ planteamientos que condividen la interacción económica como foco de estudio. Asimismo, el institucionalismo, el neo-institucionalismo y la ciencia política han contribuido sobremanera al estudio de la CTF, focalizando su atención en el rol desempeñado por políticas e instituciones. Por último, la CTF se ha planteado desde la perspectiva cultural-social constructivista, cuya idea base considera a fronteras, regiones e identidades fronterizas como espacios socialmente construidos, constantemente remodeladas por las percepciones cambiantes del "nosotros" y del "otro" (Houtum, 2000).

\footnotetext{
${ }^{10}$ El despliegue de la CTF personificada en las Euroregiones comienza al crearse la "Euroregio Gronau-Emschede" (1958), por iniciativa de los municipios de la frontera germano-holandesa, seguida de la creación de la "Regio Basilensis" (1963) a instancias de los agentes económicos y sociales de Basilea (Suiza), Mulhouse (Francia) y Friburg (Alemania).

11 Proyectos que abarcan una amplia gama temática: ordenación del territorio, desarrollo económico, transporte e infraestructura, turismo, medio ambiente, mercado laboral, sanidad, servicios sociales, cultura o desarrollo rural.

12 Donde las fronteras son percibidas como obstáculos a la eficiencia económica.
} 
Posiblemente, la definición más aceptada de cooperación transfronteriza sea la ofrecida por Perkmann (2003) $)^{13}$ que gira en torno a las interacciones entre administraciones regionales-locales fronterizas. El consenso acerca de Perkmann obedece a que la definición presenta además de un gran poder descriptivo-explicativo, grandes dosis de realidad dado que la CTF, para poder funcionar requiere, siguiendo a Morata y Noferini (2014), de un entramado relacional complejo donde confluyan tanto flujos de nivel vertical (producto de la división de funciones entre diferentes niveles institucionales ${ }^{14} \mathrm{y}$ de la interacción entre los mismos) como flujos de nivel horizontal (dada la participación de los distintos actores territoriales y de sus interacciones).

Esa complejidad necesita de la denominada gobernanza multinivel, para su correcto encauzamiento; aun tratándose de un concepto "mediático" y multireiterado (Bache, 1998; 2008), no deja de ser complejo (Marks y Hooghe, 2004) ni está exento de polémica. Por cuanto que, además de presentar notorias carencias científicas (Rosamond, 2010), subestima el poder real del Estado que sigue siendo el "guardián de la puerta" tanto en la definición de políticas como en la toma de decisiones. Si bien, permita la participación de las autoridades locales en ámbitos, anteriormente, de exclusiva competencia estatal. Aun cuando dicha participación no afecte significativamente a los resultados de la acción política estatal (Bache, 2008).

\section{Instituciones transfronterizas luso-hispanas: Comunidades de trabajo y organismos conexos}

\section{El contexto normativo europeo: Instrumentos comunitarios y no comunitarios}

Las instituciones transfronterizas luso-hispanas hunden sus raíces en el marco normativo transfronterizo europeo. Si bien, se trata de un marco peculiar puesto que los instrumentos normativos fundamentales que regulan la CTF no provienen de la Unión Europea y por lo tanto no son Derecho comunitario. En este sentido, el Convenio-Marco Europeo sobre cooperación transfronteriza entre comunidades o autoridades territoriales (1980), también denominado Tratado de Madrid (1980), ${ }^{15}$ es un tratado internacional promovido por el Consejo de Europa ${ }^{16}$ que define el marco regulador de la CTF en Europa, estableciendo

\footnotetext{
${ }^{13}$ CTF definida como "una colaboración entre autoridades subnacionales más allá de los límites fronterizos nacionales" (Perkmann, 2003, p. 157).

${ }^{14}$ Por cuanto que las autoridades locales carecen de capacidad jurídica a efectos de derecho internacional, por lo que no pueden suscribir tratados internacionales, necesitando en todo momento la cobertura de su Estado de pertenencia.

15 Tratado en vigor desde el 22 de diciembre de 1981. Suscrito por España el 1 de octubre de 1986 y ratificado el 10 de julio de1990. Hasta la fecha, 40 Estados miembros del Consejo de Europa, sobre 47, han suscrito dicho Convenio-Marco Europeo y 38 lo han ratificado.

${ }^{16}$ El Consejo de Europa (1949), promueve, mediante la cooperación de los estados europeos, la configuración de un espacio político y jurídico común en Europa sustentado sobre los valores de la democracia, los derechos humanos y el imperio de la ley. Es la más antigua de las organizaciones que persigue el ideal de la integración europea.
} 
los conceptos de "cooperación transfronteriza"17 y "comunidades o autoridades territoriales"18 (Ministerio de Hacienda y Administraciones Públicas del Reino de España [MINHAP], 2014).

A pesar de constituir un gran avance, el Tratado de Madrid presentaba una serie de limitaciones que dificultaban el funcionamiento de la CTF en Europa. Por una parte, requería la previa celebración de tratados internacionales bilaterales o multilaterales que desarrollaran y especificaran las disposiciones del Convenio-Marco Europeo (1980). Por otra, no permitía la participación de los Estados miembros (como tales), solamente de las administraciones territoriales, locales y regionales. Por último, limitaba la cooperación a entidades fronterizas contiguas o colindantes, siempre entre Estados miembros del Consejo de Europa.

Transcurridas más de tres décadas desde su aparición, se ha intentado desde el Consejo de Europa actualizar paulatinamente el Convenio-Marco Europeo (1980) mediante protocolos adicionales, el número 1 (Additional Protocol to the European Outline Convention, 1995) ha precisado, como requisito esencial de la CTF, que esta se realice por entidades que comparten una misma frontera, aunque no delimita los contenidos temáticos de dicha cooperación. Además, se posibilita la creación de organismos CTF. A su vez, el Protocolo Adicional No. 2 (Protocol No. 2 to the European Outline Convention, 1998), incorpora la denominada cooperación interterritorial,,$^{19}$ mientras que el No. 3, (Protocol No. 3 to the European Outline Convention, 2009), se orienta a facilitar la creación de agrupaciones eurorregionales de cooperación (Beltrán, 2007).

Como instrumento de Derecho comunitario en el ámbito de la CTF encontramos al Reglamento (CE) n ${ }^{\circ} 1082$ (2006) sobre la Agrupación Europea de Cooperación Territorial (АЕCT) que da origen a la misma. Entidad que, al estar dotada de personalidad jurídica permite superar las limitaciones ya reseñadas del Tratado de Madrid (1980). ${ }^{20}$ Posibilitándose fórmulas de cooperación más flexibles, adaptadas a las actuales circunstancias globales, ampliando los límites de la CTF tradicional y abarcando nuevas modalidades como la denominada cooperación territorial, como la cooperación transnacional e interregional (MINHAP, 2014).

Con la reforma de la AECT en el año $2013,{ }^{21}$ por una parte, se perfecciona el instrumento ampliando sus campos temáticos (incorporando la planificación estratégica y la gestión de cuestiones regionales y locales), admitiéndose la participación en una

\footnotetext{
17 Definida en el art. 2 como "toda acción concertada tendente a reforzar y a desarrollar las relaciones de vecindad entre comunidades o autoridades territoriales pertenecientes a dos o varias partes contratantes, así como a la conclusión de los acuerdos y arreglos convenientes a tal fin" (Convenio-Marco Europeo, 1990, art. 2.1, p. 30271).

${ }^{18}$ Define las comunidades o autoridades territoriales como "organismos que ejercen funciones locales o regionales y que son consideradas como tales en el derecho interno de cada Estado" (Convenio-Marco Europeo, 1990, art. 2.2, p.30271).

19 Definida como "cualquier concertación dirigida a establecer relaciones entre entidades territoriales de dos o más partes, distintas de las relaciones de cooperación transfronteriza entre entidades vecinas, inclusive la celebración de convenios con entidades territoriales de otros Estados" (Protocol No. 2 to the European Outline Convention, 1998, art. 1).

${ }^{20}$ Así, la AECT puede estar formada bien por Estados miembros, bien por autoridades regionales, autoridades locales y organismos regidos por el Derecho público, pertenecientes a por lo menos dos Estados miembros de la Unión Europea.

${ }^{21}$ Mediante el Reglamento (UE) n. 1302 (2013) del Parlamento Europeo y del Consejo, por el que se modifica el Reglamento (CE) n. ${ }^{\circ} 1082 / 2006$ de 5/07/2006 sobre Agrupación Europea de Cooperación Territorial dOUE n. ${ }^{\circ}$ L 210, de 31.07.2006, pp. 19-24.
} 
AECT de miembros de terceros países que sean vecinos de un Estado miembro y de los denominados países y territorios de ultramar (PTU). ${ }^{22}$

\section{Protocolos de cooperación transfronteriza y comunidades de trabajo, origen y configuración}

La institución más significativa en el panorama de la cooperación transfronteriza luso-hispana ha sido, sin lugar a dudas, la denominada comunidad de trabajo (ст), a diferencia de otras fronteras comunitarias donde la Euroregión ha tenido el protagonismo. En La Raya, la ст ha sido el instrumento de canalización sobre el terreno de los estímulos económicos aportados por la UE. Lo que la ha convertido en un ente estratégico en el despliegue operativo de la CTF luso-hispana, a pesar de las limitaciones funcionales derivadas de su naturaleza "política", de su carácter informal y de su falta de personalidad jurídica (Ponte, 2001).

Las CT luso-hispanas derivan de los Protocolos de Cooperación Transfronteriza (PCT) celebrados al amparo del Tratado de Madrid. Se trata de acuerdos suscritos entre autoridades regionales lusas fronterizas (es decir, las Comisiones de Coordinación Regionales) ${ }^{23}$ y sus contrapartes hispánicas (las comunidades autónomas espanolas [CCAA] fronterizas con Portugal) ${ }^{24}$ Los PCT constituyen acuerdos puntuales de carácter No normativo $^{25}$ (Caballero, 2009; Ponte, 2001), aunque sí que suponen un compromiso mútuo ${ }^{26}$ por parte de las regiones firmantes de avanzar en la CTF.

Las Cт luso-hispanas han orientado su actividad a la selección y seguimiento de proyectos en el marco de programas e iniciativas comunitarias, tipo INTERREG, en sus diversas fases. Incentivando las interacciones entre ambos lados de la frontera en perspectiva multinivel: desde administraciones regionales y locales, universidades, centros de estudio e investigación hasta asociaciones empresariales, profesionales y juveniles. Para la realización de dichas actividades las ст hispano-lusas se han dotado de una estructura orgánico-funcional más o menos estandarizada y de instrumentos de gestión específicos, destacando los denominados Gabinetes de Iniciativas Transfronterizas (GIT)

La ausencia de personalidad jurídica de las Ст luso-hispanas provocará toda una serie de problemas que, dada la importancia de su labor, deberán ser afrontados. De hecho, los agentes transfronterizos luso-hispanos demandarán constantemente una clarificación de dicha situación de ambigüedad jurídica, con la finalidad de lograr mayor operatividad y eficacia en el desarrollo de la CTF (Ponte y Pueyo, 2006, p. 151).

\footnotetext{
${ }^{22}$ Enumerados en el anexo II del Tratado de Funcionamiento de la Unión Europea TFUE.

${ }^{23}$ Es decir, las Regiones de norte a sur: Norte de Portugal, Región Centro de Portugal, Región del Alentejo y Región del Algarve.

${ }^{24}$ Es decir, las cCAA fronterizas con Portugal, de norte a sur: Galicia, Castilla y León, Extremadura y Andalucía.

${ }^{25}$ Por tanto, los PCT constituyen bien meras declaraciones de intenciones entre las partes firmantes, bien simples "pactos entre caballeros" que no modifican el régimen de competencias previo de que cada región suscriptora disponía anteriormente. Régimen definido por el Derecho español o por el portugués.

${ }^{26}$ Sentando las bases que posibiliten la creación de órganos e instituciones CTF, funcionales, estables, orientados a la elaboración y ejecución de programas y proyectos de interés común.
} 
Asimismo, dada la diferente regulación y estructura de las administraciones territoriales en ambos países ibéricos (fruto de la diferente comprensión del Estado) ${ }^{27}$ y visto el enorme diferencial de competencias entre las comunidades autónomas españolas y sus contrapartes portuguesas, se hacía imprescindible una urgente labor de armonización.

Para dar respuesta a tales problemas, se concluye entre España y Portugal ${ }^{28}$ el Tratado de Valencia (2004) ${ }^{29}$ que va a proporcionar cobertura jurídica a comunidades y grupos de trabajo, estableciendo tanto el derecho aplicable a los PTF como la regulación aplicable a las instituciones CTF, distinguiendo entre organismos con personalidad jurídica (como las Asociaciones de Derecho Público y Empresas Intermunicipales en Portugal o los Consorcios en el caso español) y organismos sin personalidad jurídica (comunidades de trabajo y grupos de trabajo).

Dicha distinción es significativa por cuanto que asigna áreas temáticas funcionales muy determinadas; así, los organismos sin personalidad jurídica solo podrán dedicarse a un ámbito temático restringido. ${ }^{30}$ Mientras que los organismos con personalidad jurídica pueden tener diferentes finalidades como la "realización de obras públicas, la gestión común de equipamientos o servicios públicos, y el desarrollo de las acciones que les permitan beneficiarse de fondos europeos" (MINHAP, 2014, p. 5).

La cobertura jurídica de lo realizado por comunidades y grupos de trabajo hasta la entrada en vigor del Tratado de Valencia (2004) supondrá necesariamente la adaptación de los Protocolos de Cooperación Transfronteriza (PCT), preexistentes a la nueva regulación, lo que se realizará mediante la figura del convenio de adaptación. ${ }^{31}$

\section{Aspectos organizativos, administrativos y funcionales de las comunidades de trabajo luso-hispanas}

Con la constitución de la Comunidad de Trabajo Galicia-Norte de Portugal del 31 de octubre de 1991, se inicia el proceso de institucionalización de la incipiente CTF luso-hispana. Al ser la primera, la CT Galicia-Norte de Portugal se configuró como modelo, para bien o para mal de las restantes (Ponte y Pueyo, 2006). De ahí que, convenga detenerse someramente en sus órganos de gestión: presidencia, consejo, comité de coordinación y comités sectoriales, puesto que con variantes, estos se han

\footnotetext{
${ }^{27}$ En el caso luso, un país tremendamente centralizado. Mientras que el caso hispano aparece como paradigma de descentralización.

${ }^{28}$ Antecedente del Tratado de Valencia (2004) es el Tratado de Amistad y Cooperación entre España y Portugal (1977), suscrito simultáneamente a la incorporación de España al Consejo de Europa. Dirigido a fortalecer los vínculos de amistad y solidaridad luso-hispanos.

29 Tratado bilateral entre el Reino de España y República Portuguesa sobre Cooperación transfronteriza entre entidades e instancias territoriales firmado en Valencia el 3 de diciembre de 2002, y que entró en vigor el 30 de enero de 2004.

${ }^{30}$ Es decir, al "estudio de cuestiones de interés mutuo", la "formulación y realización de propuestas de cooperación", la "preparación de estudios, planes, programas y proyectos", la "promoción de formas de relación entre los agentes de este tipo de cooperación", y la "ejecución de las tareas previstas para estas estructuras" en el Programa Interreg (MINHAP, 2014, p. 5).

${ }^{31}$ Como por ejemplo, el Convenio de Cooperación Transfronteriza de adaptación del Protocolo de Cooperación entre la Comunidad de Castilla y León y la Comisión de Coordinación de la Región Centro de Portugal del 3 de marzo de 1995, al Tratado de Valencia suscrito el 18 de noviembre de 2008.
} 
replicado en las restantes comunidades de trabajo (Ст). La presidencia, ${ }^{32}$ se ejercía alternativamente durante dos años por los presidentes de las respectivas regiones miembros. El consejo, órgano deliberante de carácter plenario, se reúne dos veces al año, debiendo dar continuidad a las actividades de CTF, fijando el "Programa de acción" de la CT y estableciendo sus normas de funcionamiento. El consejo está integrado por las delegaciones oficiales de la Comunidad Autónoma de Galicia y de la Comisión de Coordinación de la Región Norte de Portugal, en las cuales debían participar en la medida de lo posible, los representantes de las colectividades subregionales transfronterizas, así como los coordinadores de las comisiones sectoriales.

A su vez, el comité de coordinación asume las funciones de coordinar las comisiones sectoriales, del funcionamiento de la secretaría, del seguimiento del "Programa de acción" y de la elaboración de los "Programas de trabajo" de la ct (Ponte y Pueyo, 2006).

Por último, las comisiones sectoriales paritarias se encargaban de los asuntos de interés común para las partes: administraciones regionales y locales; agricultura, medio ambiente, recursos naturales y ordenación territorial; cultura, patrimonio y turismo; desarrollo local; dinamización económica; enseñanza, formación y empleo; investigación científica y universidades; pesca; sanidad y asuntos sociales; y transportes (Ponte y Pueyo, 2006).

Característica específica de los denominados grupos de trabajo Extremadura-Alentejo y Extremadura-Centro es que las funciones del consejo y del comité de coordinación las asume el propio grupo de trabajo, actuando como órgano responsable de la coordinación y de la planificación general de los trabajos de cTF. Asistiendo, al menos, dos veces al año, a reuniones que son presididas por los máximos responsables políticos en materia de cooperación transfronteriza. El grupo de trabajo está integrado por los responsables regionales de cada una de las comisiones específicas o técnicas (las denominadas "sectoriales" en la Comunidad de Trabajo Galicia-Norte de Portugal) ( $E l$ Gabinete de Iniciativas Transfronterizas, s.f.).

Las comunidades de trabajo Castilla y León-Norte de Portugal y Castilla y León-Región Centro (y por extensión, las dos andaluzas) seguirán un organigrama standard: consejo plenario (el "parlamento" de la comunidad de trabajo) con funciones, de establecer y aprobar tanto el programa de acción general (propuesto por el presidente) como el informe general bianual de actividades, regular el funcionamiento de los comités sectoriales y otros órganos auxiliares, etcétera (Junta de Castilla y León, s.f.).

Por su parte, el consejo directivo (una suerte de "gobierno" ejecutivo) se encarga de coordinar las actividades de la comunidad de trabajo, asegurando la continuidad de sus trabajos, elaborando los programas de acción y el informe bianual de actividades. Asimismo, realiza el seguimiento, evaluación y control de las actividades del plan de acción aprobado por el consejo plenario y llevar a cabo las tareas de ejecución encomendadas por dicho órgano. Igualmente, dirige la actividad y el funcionamiento de la secretaría (Gabinete de Iniciativas Transfronterizas [GIT]) (Junta de Castilla y León, s.f.). Por último, el consejo directivo asigna a los comités sectoriales el estudio y análisis de problemas transfronterizos del ámbito material que corresponda y la elaboración de propuestas de solución.

${ }^{32}$ Figura que ostentaba la representación del organismo, convocaba y presidia el Consejo y ratificaba sus decisiones. 
Resulta evidente el protagonismo alcanzado por los GIT, órganos de carácter técnico-administrativo, orientados a garantizar flujos de información estables y contactos permanentes entre las diversas instituciones que configuran las comunidades de trabajo, gestionando las actividades y el día a día de la cooperación transfronteriza. Fue el grupo de Trabajo Extremadura-Alentejo el primero en introducir la figura del Gabinete de Iniciativas Transfronterizas (GIT), de hecho, sus buenos resultados provocaron la creación del GIT Extremadura-Región Centro, extendiéndose dicha figura a las restantes Comunidades de Trabajo ibéricas (El Gabinete de Iniciativas Transfronterizas, s.f.).

Además de sus funciones de secretariado, los diversos GIT se han destacado en sus diversos ámbitos territoriales por ser grandes dinamizadores y catalizadores de la CTF, estimulando todo tipo de relaciones y proyectos entre ambos lados de La Raya, apoyando la realización de cursos, jornadas y seminarios, la celebración de encuentros transfronterizos de temática variada (profesionales, técnicos, comerciales, lúdicos y ferias), la edición y difusión de publicaciones, entre otras. Asimismo, los GIT gestionan proyectos propios que estimulan la cooperación territorial, no sólo a nivel transfronterizo sino también a nivel interregional (Gabinete de Iniciativas Transfronterizas de Castilla y León, 2014).

\section{La evolución del modelo CTF luso-hispano: De las comunidades de trabajo a las Euroregio}

La evolución del modelo de CTF luso-hispano "tradicional", organizado en torno a comunidades y grupos de trabajo, presenta dos líneas evolutivas muy definidas. Por una parte, una tendencia continuista del formato sustentado en los Protocolos de Cooperación (adaptados sucesivamente al Tratado de Valencia, 2004), encarnado en las denominadas Comunidades de Trabajo Tripartitas. Por otra, una tendencia rupturista-innovadora personificada por la Agrupación Europea de Cooperación Territorial denominada "Galicia-Norte de Portugal" (GNP-AECT), que constituye un cambio sustancial, puesto que al fundamentarse en el Reglamento Europeo (Reglamento [CE] $\mathrm{n}^{\circ}$. 1082, 2006), como tal AECT dispone de personalidad jurídica propia e independiente y, además, de capacidad administrativa propia: ${ }^{33}$ pudiendo diseñar, proyectar, presentar ante las instancias comunitarias programas y proyectos y ejecutarlos de forma independiente, sin necesidad de que tales programas sean validados o supervisados por los gobiernos español o portugués.

La línea continuista es la elegida por las entidades regionales luso-hispanas de la mitad centro-sur (es decir, las CCCAA de Andalucía y Extremadura y las CCDR portuguesas homólogas, Alentejo, Centro y Algarve), interesadas en profundizar sus relaciones CTF, dados los buenos resultados obtenidos ${ }^{34}$ con el modelo tradicional de cooperación, si bien simplificándolo, racionalizando estructuras organizativas, procedimientos y dota-

\footnotetext{
${ }^{33}$ Por lo que puede "licitar, contratar y realizar obras públicas, expropiar, realizar contratos, gestionar en común equipamientos y explotar servicios de interés general" (Cancela, 2011, p.12).

${ }^{34}$ Las infraestructuras realizadas han mejorado enormemente la permeabilidad de La Raya. Igualmente, las transacciones comerciales y las relaciones entre empresas se intensifican constantemente. También parece haber mejorado el conocimiento cultural del "otro" mediante el incremento de la enseñanza del portugués en Extremadura y Andalucía y del español en Algarve, Alentejo y Centro.
} 
ciones presupuestarias. Para ello se firma un único convenio de cooperación (tripartito), simplificándose las cuatro preexistentes Comunidades de Trabajo en solo dos. Ambos Convenios Tripartitos de CTF van a dar lugar a las Euroregiones "Alentejo-Algarve-Andalucía" (EUROAAA) y "Alentejo-Centro-Extremadura" (EUROACE). En ambos casos, se constituye un organismo sin personalidad jurídica, cuya forma jurídica es la de "Comunidad de Trabajo" (regulada por el Tratado de Valencia, 2004).

Por lo que respecta a la línea rupturista, las intensas relaciones transfronterizas ya existentes aconsejaban reforzar la institucionalidad preexistente constituyéndose la AECT "Galicia-Norte de Portugal", cuyo objetivo es facilitar y fomentar la cooperación territorial (transfronteriza, transnacional o interregional) entre sus miembros, con el fin exclusivo de reforzar la cohesión económica y social. La Euroregión Galicia-Norte de Portugal naciente presenta una estructura compleja de un lado la Comunidad de Trabajo Galicia-Norte de Portugal subsiste configurada como órgano político, vínculo orgánico entre la GNP-AECT y las máximas instancias políticas regionales. Del otro lado, la GNP-AECT, "ejecutor" de los programas transfronterizos (Cancela, 2011). Como nexo de articulación entre ambas se establece la figura jurídica del Consejo Superior, integrado por los coordinadores generales de la cT en representación de la Xunta de Galicia y la Comissão de Coordenação e Desenvolvimento Regional do Norte (CCDR-N).

\section{Desactivación fronteriza versus no desactivación en la frontera luso-hispana}

La profusión de iniciativas CTF desde 1991 ¿Ha contribuido a eliminar o minimizar la suma de los efectos frontera y callejón sin salida? y, en definitiva ¿Se han reducido sustantivamente los costes de transacción en la frontera hispano-lusa? En línea de máxima, la heterogeneidad y el claroscuro de situaciones planteadas a lo largo de La Raya no permite una respuesta unívoca, por lo que hemos de retrotraernos a las recomendaciones ${ }^{35}$ planteadas por la ARFE para el conjunto de La Raya, en la década de los noventa, para tratar de comprender las luces y sombras presentes en el proceso desactivador.

Dichas recomendaciones enfatizaban, sobremanera, las grandes carencias existentes en La Raya en lo que se refiere a infraestructuras y equipamientos. El énfasis en tales dotaciones, parece haber provocado una respuesta reactiva de acentuado sesgo "viario" y constructor por parte de las instituciones transfronterizas luso-hispanas ya en el diseño, ya en la aplicación de los programas comunitarios en La Raya ibérica.

Siguiendo dichas coordenadas, se observan grandes avances en términos de dotaciones de infraestructuras viarias y de grandes redes de transporte y comunicación a lo largo de toda la extensión de La Raya. Esta nítida mejora en infraestructuras viarias ha impulsado extraordinariamente el volumen de los tráficos fronterizos (flujos de mercancías, personas, vehículos, inversiones, etcétera) a través de los principales ejes

\footnotetext{
${ }^{35}$ Para las regiones fronterizas de Portugal y de España las barreras siguen siendo una infraestructura aún por mejorar así como la falta de posibilidades de comunicación transfronteriza. Debido al retraso estructural y a la posición geográfica en la periferia, es particularmente difícil explotar las ventajas del mercado interior ampliado de la UE, por ejemplo en los intercambios transfronterizos, en el mercado laboral transfronterizo o en las condiciones de establecimiento ventajosas para los nuevos inversores (ARFE, 1997, p. 18).
} 
viarios fronterizos ${ }^{36}$ como muestra la Tabla 1. Evidentemente, la incorporación al Espacio Schengen de ambos países ibéricos ${ }^{37}$ (1995) con la subsiguiente desaparición de controles y aduanas, ha redundado en la gran expansión mercantil observada.

Tabla1: Intensidad de tráficos viarios en los tramos fronterizos luso-hispanos

\begin{tabular}{|c|c|c|c|}
\hline Eje viario & Tipología & \multicolumn{2}{|c|}{ Tráficos } \\
\hline $\begin{array}{l}\text { Tramo Galicia-Región Norte } \\
\text { de Portugal }\end{array}$ & & Portugal & España \\
\hline $\begin{array}{l}\text { Tuy (Puente nuevo) España/Valença do } \\
\text { Minho (Portugal) }\end{array}$ & $\mathrm{A}-55 / \mathrm{IP}-1 \mathrm{~A}$ & 14.725 & 15.511 \\
\hline Tuy (Puente viejo)/Valença do Minho & N-550 / EN-13 & & 4.903 \\
\hline Arbo/Melgaço & PO -405 PO- $400 \mathrm{~b}$ & & 1.279 \\
\hline Ponte Barxas/San Gregórico & OU-801 (ou-410) & & 1.433 \\
\hline Verín/Vilaverde Raia & N-532/ EN-103 (IP-3) & 4.094 & 4.555 \\
\hline \multicolumn{4}{|l|}{$\begin{array}{l}\text { Tramo Castilla-León/Región Centro } \\
\text { de Portugal }\end{array}$} \\
\hline San Martín de Pedroso/Quintanilla & N-122/EN-218-1 (IP-4) & 1.377 & 1.564 \\
\hline La Fregeneda/Barca de Alva & CL-517 /ER-221 & & 1.070 \\
\hline Fuentes de Oñoro/Vilar Formoso & N-620 / IP-5 & 7.739 & 6.676 \\
\hline \multicolumn{4}{|l|}{$\begin{array}{l}\text { Tramo Extremadura/Región Centro } \\
\text { de Portugal-Alentejo }\end{array}$} \\
\hline Valverde del Fresno/Penamacor & EX-205/ & & 1.287 \\
\hline Valencia de Alcántara/Marvao & N-521 / EN-246-1 & & 1.043 \\
\hline Badajoz/Caia (Elvas) & $\mathrm{N}-\mathrm{V} / \mathrm{IP}-7 \mathrm{~A}$ & 9.426 & 8.669 \\
\hline Olivenza/Ajuda & EX-105/ & & 958 \\
\hline Villanueva del Fresno/Leonardo (Mourao) & EX-107/ EN-256 & & 1.419 \\
\hline \multicolumn{4}{|l|}{ Tramo Andalucía-Algarve-Alentejo } \\
\hline Rosal de la Frontera/Vila V. Fícalho & N-433/ EN-260 (IP-8) & & 1.587 \\
\hline $\begin{array}{l}\text { Ayamonte/Monte Francisco (Vila Real de } \\
\text { Santo António) }\end{array}$ & A-49 / IP-1 A / & 6.788 & 9.706 \\
\hline
\end{tabular}

Fuente: Elaboración propia, datos provenientes de Díaz (2007, pp. 299-300).

El hecho de que la mitad de los ejes viarios de mayor tráfico se localicen en el tramo Galaico de La Raya, entre las regiones de Minho (Portugal) y Galicia (España), evidencia hasta qué punto las interacciones transfronterizas son particularmente intensas en este tramo fronterizo.

\footnotetext{
${ }^{36} \mathrm{Si}$ bien, solo seis grandes ejes concentran $81 \%$ del volumen del tráfico transfronterizo hispano-luso: Tuy (Puente nuevo)/Valença do Minho (25\%); Tuy (Puente viejo)/Valença do Minho (8\%); Verín/Vilaverde Raia (7\%); Fuentes de Oñoro/Vilar Formoso (11\%); Badajoz/Caia-Elvas (14\%) y Ayamonte/Monte Francisco-Vila Real de S. Antonio (16\%). Normalmente se trata de Autopistas o Autovías (antiguas carreteras "nacionales", desdobladas).

${ }^{37}$ Aunque Portugal y España se adhieren al Tratado de Shengen en 1991, hasta marzo de 1995 no entró en vigor el Convenio de aplicación de dicho acuerdo.
} 
Asimismo, se observa una gran intensidad de tráfico tanto en el eje Badajoz-Elvas (en el tramo extremeño de La Raya entre Extremadura y el Alentejo) como en el eje Ayamonte-Vila Real de Santo Antonio localizado en el tramo sureño de la frontera (entre el Algarve, Portugal y Andalucía, España).

Dado el diferencial de dotaciones viarias existente entre 1991 y la actualidad, resulta lógico que se dé, una suerte de consenso, en torno a la mejora de los transportes y las comunicaciones en La Raya ibérica (Caballero, 2009; Gutiérrez, Pérez y Mora, 2010; Martín, 2012; Medina, 2007; Rosell, 2012). Sin embargo, existen voces disidentes al respecto: para ciertos autores la influencia de los Fondos Estructurales y sus consecuencias viarias e infraestructurales se ha sobreestimado (al menos en sus primeras fases), por cuanto que su existencia, a todas luces buena, bien incorpora importantes costes de conservación y mantenimiento (Cobo, 1999), bien su impacto resulta relativamente débil en el territorio fronterizo (Caballero, 2009), dadas sus carencias estructurales.

Para otros autores, si bien el desarrollo viario resulta evidente, parece mucho más orientado a la conexión estatal "macro" Portugal/España que a la articulación fronteriza rayana "micro" entre ambos lados de la frontera (Márquez, 2012). Con ello se ha generado una dinámica espacial dual, ya que al concentrarse la acumulación de infraestructuras en los cinco grandes ejes viarios ${ }^{38}$ citados (Tabla 1) y en la malla de transportes conectada a estos, la mayor parte del espacio fronterizo rayano presenta evidentes carencias viarias y de servicios de transporte (Gutiérrez et al., 2010, p. 144).

Efecto colateral de la concentración de infraestructuras y equipamientos es el reforzamiento de la tendencia a la distribución asimétrica poblacional. Así, la población rayana tiende a concentrarse en los emplazamientos mejor comunicados y equipados que permiten una mayor calidad de vida (mejores expectativas de empleo, educación, etcétera). Con ello tiende a debilitarse, aún más, el poblamiento de las áreas rurales peor comunicadas, agravándose tanto la regresión demográfica como el proceso de envejecimiento ya en acto (Lois y Carballo, 2015; Podadera y Calderón, 2014).

La intensificación de los tráficos fronterizos aparece directamente correlacionada al incremento sustantivo de las interacciones comerciales, inversoras y financieras luso-hispanas. Multiplicándose los contactos y la presencia empresarial tanto por parte española como portuguesa, a uno y otro lado de la frontera, teniendo como resultado la duplicación de cifras y volúmenes de importaciones y exportaciones (Alonso, 2009; Lucio y Barrios, 2002; Medeiros, 2010 y 2015; Pires y Teixeira, 2002, 2003).

Resulta especialmente significativo el incremento del tráfico comercial entre regiones fronterizas, especialmente intenso entre Galicia y la Región Norte (Díaz, 2007) y en el eje Badajoz/Elvas-Campo Maior. Tráfico que supone $60 \%$ de la exportación pacense y $50 \%$ de su importación. En el tramo sur de la frontera, el comercio entre Andalucía con sus homólogas fronterizas, Algarve (PT) y Alentejo (PT), ha convertido a Portugal en el segundo destino de las exportaciones andaluzas ${ }^{39}$ (Gómez y Méndez, 2012).

\footnotetext{
${ }^{38}$ La acumulación se produce tanto en los grandes ejes transfronterizos como en los grandes núcleos urbanos atravesados o vinculados por tales ejes.

${ }^{39}$ Aproximadamente 1500 empresas andaluzas exportan sus productos a Portugal, hecho inédito en términos históricos. Asimismo, los empresarios lusos están muy presentes en Andalucía, contando con centros logísticos en Sevilla.
} 
A pesar del aumento exponencial (en términos históricos) de inversiones, flujos de capital y personas ya constatado que podrían hacernos pensar que aquella histórica frontera económico-comercial-aduanera luso-hispana ya no existe, continúan muriendo en la frontera importantes equipamientos como las redes eléctricas, telefónicas, energéticas, financieras, los servicios médicos, sociales y asistenciales (Gutiérrez et al., 2010; Medina, 2007).

Por lo que respecta a los costes de transacción, se siguen produciendo restricciones al libre tránsito, ya en los campos administrativos (limitaciones administrativas al transporte de mercancías, matriculaciones de vehículos, etcétera), ya en los campos fiscales (declaraciones de renta, pago de impuestos, etcétera). Asimismo, en el campo administrativo-institucional persisten las marcadas diferencias entre estructuras administrativas españolas y portuguesas. Diferencias complicadas por el diferente ordenación territorial (Gutiérrez et al., 2010, p. 145), por lo que, por ejemplo, un municipio español no coincide con un concelho portugués. ${ }^{40}$ Tampoco las provincias españolas se corresponden con los distritos portugueses y el nivel de competencia de las Comunidades Autónomas hispanas no tiene equivalente en las regioes portuguesas. Si bien, la población fronteriza no percibe dichas trabas, en su conjunto, como demasiado significativas (Gutiérrez et al., 2010).

No debería omitirse que a nivel estadístico no existe la necesaria homogeneización de cara a establecer las diferentes variables estadísticas (Gutiérrez et al., 2010, p. 145). Además, se haría necesario aproximar (o al menos coordinar) los diferentes sistemas jurídico-legislativos, puesto que la actual disparidad normativa dificulta la actuación conjunta y colaborativa luso-hispana (Gutiérrez et al., 2010, p. 146).

Sí que parece persistir, inmutable al tiempo, la frontera social-cultural e identitaria, ya bajo la forma de "sentimiento de identidad diferenciada e incluso distante" (Medina, 2007 , p. 142), ya de forma intangible, pero presente, en la mente de la gran mayoría de sus habitantes (Alonso, 2009; Antunes, 2008; Gualda, 2009; Gutiérrez et al., 2010), síntoma evidente de que la frontera luso-hispana resulta una simbólica y nítida "construcción social”.

\section{Conclusiones}

A partir de 1991 se viene produciendo un proceso de desactivación fronteriza en La Raya luso-hispana, en consonancia con su condición de frontera interior comunitaria. Proceso que se ha llevado a cabo siguiendo las coordenadas de la integración europea (vía MUE) y de sus procesos actuativos: eliminación de controles físicos, barreras y aduanas para conseguir el libre tránsito, desarrollo de la cooperación transfronteriza y aplicación de programas y fondos estructurales comunitarios, en particular Interreg.

La aplicación de dichos mecanismos (y de sus ingentes recursos financieros) ha incentivado procesos de inversión y acumulación de capital (particularmente en infraestructuras y equipamientos) que han actuado como dinamizadores económicos sobre un espacio que presentaba hasta fechas, relativamente recientes, un perfil socioeconómico próximo al subdesarrollo estructural.

${ }^{40}$ Configurado a partir de las freguesias o parroquias que lo integran. 
Por lo que respecta a la desactivación fronteriza, se constata la minimización de los costes de transacción gracias al libre tránsito existente con la desaparición de aduanas, controles y barreras, así como grandes avances en los campos de las infraestructuras viarias y de grandes redes de transporte y comunicación transfronterizas. Factores determinantes (y condicionantes) de la notable expansión observada en los tráficos de mercancías, bienes, servicios y personas. Personificados en el incremento exponencial del comercio internacional luso-hispano, con especial atención al crecimiento de las interacciones comerciales de determinadas regiones fronterizas.

A pesar de los progresos observados, subsisten todavía algunos obstáculos de relevancia de tipo administrativo, logístico y normativo para lograr la completa remoción del efecto frontera en La Raya Ibérica. Por ello, si la pregunta a responder fuera ¿Se puede desactivar el efecto frontera derivado de la existencia del confín? La respuesta en el caso de La Raya (y en otras tantas fronteras interiores europeas) sería sí, con ciertas salvedades, puesto que a pesar de los grandes progresos constatados todavía quedan algunos campos donde quedan bastantes cosas por hacer.

En dicho proceso la CTF y sus instituciones, con sus pros y contras, han desempeñado un rol fundamental en el proceso de captación y canalización territorial de los recursos financieros, de ahí que su influencia en el panorama político, administrativo y social de La Raya ibérica resulte innegable. Las instituciones CTF, especialmente las Comunidades de Trabajo y sus GIT, han generado con su actividad una nueva madeja de relaciones, interacciones e intereses comunes anteriormente inexistentes, sentando las bases de futuros y esperanzadores desarrollos conjuntos (Medeiros, 2015), iniciativas como las redes transfronterizas Resoe, ${ }^{41}$ RIET, ${ }^{42}$ el Eixo Atlántico do Noroeste Peninsular, ${ }^{43}$ etcétera (que bien pudieran ser objeto de ulteriores trabajos), inimaginables un par de décadas atrás, lo evidencian.

Si bien, queda mucho espacio para la mejora, puesto que además de tratarse de una cooperación "desde arriba" (impulsada por las autoridades regionales) y, en este sentido, posiblemente demasiado "institucional" (en ciertos aspectos incluso "burocrática"), nacida al calor de los fondos y subvenciones uE, (Calderón, 2010; Márquez, 2012; Medina, 2007). Asimismo, se ha venido implementando de forma unilateral o, si se quiere, muy poco transfronteriza, con duplicación nacional de organismos. Además ha dado prioridad a infraestructuras y comunicaciones, omitiendo aspectos sociales o culturales relevantes (Cabero, 2004, 2008; Caramelo, 2007). Por último, se trata de una cooperación "frágil", dada su dependencia casi extrema de los Fondos Estructurales.

Los progresos desfronterizadores han producido la "práctica" invisibilización de la vieja frontera, puesto que el viajero no encuentra barreras que impidan su paso, ni aduaneros que exijan documentación, ni policías que controlen los tráficos fronterizos. Solo algunos carteles recuerdan el tránsito de España a Portugal y viceversa. Por

\footnotetext{
${ }^{41}$ La denominada Regiones del Sudoeste Europeo (RESOE), una macroregión integrada por las CCAA españolas de Galicia, Castilla y León, Asturias y la región portuguesa del Norte de Portugal, el objetivo de constituir un espacio estratégico que favorezca el desarrollo económico y los reequilibrios territoriales.

42 La llamada Red Ibérica de Entidades Transfronterizas de Cooperación (RIET), integrada por organizaciones que trabajan en el ámbito de la CTF luso-hispana y que plantean esta como "todo trabajo conjunto entre España y Portugal", considerando a la cooperación como un factor determinante en el desarrollo local y regional, de los territorios fronterizos.

${ }^{43}$ Entidad integrada en la actualidad por más de una treintena de ciudades de la Euroregión GNP.
} 
lo que podría pensarse que la antigua Raya ibérica ha desaparecido. Sin embargo, la vieja frontera sigue ahí, presente, viva en la mente de la gran mayoría de sus habitantes, viva en sus dimensiones culturales, lingüísticas, emocionales e identitarias, viva en el sentimiento de pertenencia a una comunidad nacional, distinta y diversa de las restantes. Por ello, si la cuestión a responder fuera ¿Se pueden suprimir las fronteras? la respuesta en el caso de la vieja frontera hispano-lusa (y, posiblemente, de otras tantas fronteras intraeuropeas), sería no; se pueden invisibilizar pero no quitar como si nunca hubieran existido, y esa contradicción de fondo sigue latente en Europa a pesar del mue y del proceso de integración europea.

Si las fronteras intracomunitarias son fronteras en transición, para completar dicha obra se hace necesario, por una parte, que no se produzcan rebrotes nacionalistas (tentación muy presente en la Europa actual), además, de una voluntad política clara y definida al respecto. Por otra parte, para que la voluntad no se quede en mero voluntarismo es preciso dar continuidad a las iniciativas CTF. Ahí parece radicar el problema, vistos los enormes problemas económicos, los riesgos derivados y los desafíos sociales que deben gestionar tanto España como Portugal.

\section{Referencias}

Alonso, J. L. (2009). Las relaciones económicas de España y Portugal desde 1986. ¿Hacia la formación del mercado ibérico? Boletín de la Asociación de Geógrafos Españoles, (49), 129-153.

Amilhat-Szary, A. L. y Fourny, M. C. (2006). Après les frontières, avec la frontière, nouvelles dynamiques transfrontalières en Europe. La Tour d'Aigues, Francia: Editions de l'Aube.

Anderson, J., O’Dowd, L. y Wilson, T. M. (2003). New borders of changing Europe: Cross-border cooperation and governance. Londres, Reino Unido: Taylor \& Francis.

Antunes, M. D. (2008). Fronteras estatales y relaciones sociales en la frontera hispano-portuguesa. El caso de Barrancos y Oliva de la Frontera. Gazeta de Antropología, (24), 24-52.

Asociación de las Regiones Fronterizas Europeas (ARFE). (1997). Documento de Trabajo sobre la iniciativa INTERREG de la UE y sus futuros desarrollos. Recuperado de http:// www.aebr.net/publikationen/pdfs/interreg_97.es

Bache, I. (1998). The politics of European Union regional policy: Multi-level governance or flexible gatekeeping? Sheffield, Inglaterra: Sheffield Academic Press.

Bache, I. (2008). Europeanization and multi-level governance. Cohesion policy in the European Union and Britain. Lanham, Estados Unidos: Rowman \& Littlefield.

Baigorri, A. y Cortés, G. (1997). Un nuevo desafio en el análisis regional: las ciudades y regiones fronterizas. Presentado en la XXIII Reunión Española de Estudios Regionales, Valencia.

Beltrán, S. (2007). La cooperación transfronteriza e Iinterterritorial: Un clásico renovado. Revista d'estudis autonòmics i federals, (4), 215-246.

Boira, J. V. (2004). Les euroregions. Valencia, España: Institut d'Economia i Empresa Ignasi Villalonga.

Boman, J. (2005). Identity and institutions shaping cross-border cooperation: A comparative study of cross-border cooperation in the Estonian-Russian and Romanian-Moldovan borderlands (Tesis). University of Tartu, Estonia. 
Caballero, A. (2009). Fronteras compartidas. La Raya Centro-Ibérica. Revista de Estudios Extremeños, LXV (I), 417-448.

Cabero, V. (2004). Iberismo y cooperación. Pasado y futuro de la Península Ibérica. Porto, Portugal: Campo Das Letras Editores.

Cabero, V. (2008). El ámbito de cooperación transfronteriza en Castilla y León: entre la frustración y la esperanza. En L. Domínguez (Dir.), A cooperaçao transfronteiriça entre Portugal e Espanha (pp. 175-197). Vigo, España: Eixo Atlântico do Noroeste Peninsular.

Cairo, H. y Lois, M. (2013). La construcción histórico-política del territorio: El caso hispano-portugués. Historia y Política, (30), 13-21.

Cairo, H., Godinho, P. y Pereiro, X. (2009). Portugal e Espanha entre discursos de centro e praticas de fronteira. Lisboa, Portugal: Colibrí, Instituto de Estudios de Literatura Tradicional.

Calderón, F. J. (2010). La Raya luso-hispana, una frontera en transición. En J. A. Márquez (Coord.), Actas del Congreso Internacional Cooperación Transfronteriza Andalucía-Algarve-Alentejo (pp. 41-54). Huelva, España: Servicio de Publicaciones de la Universidad de Huelva.

Calderón, F. J. (2015). Repasando la frontera hispano-portuguesa: Conflicto, interacción y cooperación transfronteriza. Estudios Fronterizos, 16(31), 65-89. Recuperado de http://ref.uabc.mx/ojs/index.php/ref/article/view/241

Cancela, C. (2011). La Eurorregión Galicia-Norte de Portugal: Un escenario de cooperación territorial”. Razón y Palabra, 15(74).

Caramelo, S. (2007). Uniâo Europeia, fronteira e territorio. Porto, España: Campo das Letras.

Chen, N. (2004). Intra-national versus international trade in the European Union: Why do national borders matter? Journal of International Economics, 63(1), 93-118.

Cobo, R. (1999). La contribución de los fondos estructurales al desarrollo de la región del Duero. En La Unión Europea y los fondos estructurales en el desarrollo sostenible y la ordenación del territorio de la región del Duero (pp. 23-29). Zamora, España: Fundación Rei Afonso Henriquez.

Coletti, R. (2010). Cooperación transfronteriza y trayectorias de desarrollo: aprendizajes de la experiencia europea. Si somos americanos. Revista de Estudios Transfronterizos 10(1), 154-173.

Council of Europe. (2006). Practical guide to transfrontier cooperation. Recuperado de http:// www.espaces-transfrontaliers.org/fileadmin/user_upload/documents/Documents_MOT/Etudes_Publications_MOT/Practical_guide_COE_MOT_EN.pdf

Council of Europe. (2009). The state of transfrontier co-operation in Europe. Recuperado de https: / / wcd.coe.int/ViewDoc.jsp?p=\&id=1560077\&Site=CM\&direct=true

Díaz, J. A. (2007). Las relaciones transfronterizas Galicia-Región Norte de Portugal: Estrategias para la dinamización del potencial de desarrollo endógeno de la Eurorregión. Revista de Desarrollo Rural y Cooperativismo Agrario, (10), 291-308.

Domínguez, L. y Varela, E. J. (2015). Construyendo "Europolis" a partir de la cooperación local "informal" en los espacios transfronterizos: el caso de la RIET. Geopolitica, 6(2), 225-246.

Dörrenbacher, H. P. y Brücher, W. (2000). Espace transfrontalier et culture mixte: l'exemple sarro-lorrain. Géographie et cultures, (36), 57-70. 
Dupeyron, B. (2008). L'Europe au défi de ses frontières: expériences rhénane et pyrénéenne, Berna, Suiza: PIE, Peter Lang.

El Gabinete de Iniciativas Transfronterizas. (s.f.). Recuperado de http://alcazaba.unex. es/ emarnun/gabi/index.htm

Evans, C. L. (2003). The economic significance of national border effects. American Economic Review, 93(4), 1291-1312.

Farinós, J. y Payà, M. (Septiembre de 2005). Cooperación territorial transfronteriza y cohesión territorial. España y Portugal ante la futura política regional europea. Actas X Colóquio Ibérico de Geografia: "A Geografia Ibérica no Contexto Europeu”, Universidade de Évora, Portugal. Recuperado de http://www.apgeo.pt/files/ docs/CD_X_Coloquio_Iberico_Geografia/pdfs/005.pdf

Fernández, P. A. (2008). Aspectos generales de la cooperación transfronteriza en el marco europeo. En P. A. Fernández (Dir.), La asimetría institucional entre España y Portugal en el marco de la cooperación transfronteriza (Andalucía, Algarbe y Alentejo) (pp. 61-76). Barcelona, España: Atelier.

Foucher, M. (2007). L'obsession des frontiers. París, Francia: Perrin.

Gabbe, J. (2004). The Euroregion as a place for transfrontier cooperation implementation. Quarterly of International Sociology Cooperation and Euroregions, XIII(3-4), 30-52.

Gabinete de Iniciativas Transfronterizas de Castilla y León. (2014). Publicaciones GIT de Castilla y León. Valladolid, España: Consejería de la Presidencia, Junta de Castilla y León.

Gildersleeve, C. R. (1976). The status of borderlands studies. Geography. Social Science Journal, 13(1), 19-28.

Gil-Pareja, S., Llorca-Vivero, R., Martínez-Serrano, J. A. y Oliver, J. (2005). The border effect in Spain. The World Economy, 28(11), 1617-1631.

Gómez, J. M. y Méndez, S. (2012). Identidad cultural de la eurorregión Andalucía/ Algarve/Alentejo: Tratamiento informativo en la prensa de Sevilla. Razón y Palabra, (80).

Gualda, E. (2009). Realidad social en Andalucía, Algarve y Alentejo. Huelva, España: Servicio de Publicaciones de la Universidad de Huelva.

Gutiérrez, J. A., Pérez, J. M. y Mora, J. (2010). Dimensión y tipología de los movimientos transfronterizos en la frontera entre España (Extremadura) y Portugal (Alentejo y Región Centro). Documents d’Anàlisi Geogràfica, 56(1), 133-148.

Harguindéguy, J. B. (2007). La frontière en Europe: un territoire? Coopération transfrontalière franco-espagnole. París, Francia: L'Harmattan.

Heredero, M. I. y Olmedillas, B. (2009). Las fronteras españolas en Europa: de INTERREG a la cooperación territorial europea. Investigaciones Regionales, (16), 191-215.

Houtum, H. van (2000). An overview of European geographical research on borders and border regions. Journal of Borderland Studies, XV(1), 57-83.

Houtum, H. van y Strüver, A. (2002). Borders, strangers, doors and bridges. Spaces $\mathcal{E}^{\circ}$ Polity, 6(2), 141-146.

Julio, M. C. (2012). Hacia una cooperación transfronteriza efectiva en la frontera colomboecuatoriana (Tesis de maestría). Universidad de Salamanca, Instituto de Iberoamérica, España. Recuperado de http://gredos.usal.es/jspui/bitstream/10366/120168/1/ TFM_Estudioslatinoamericanos_\%20Julio_Carmela.pdf

Junta de Castilla y León. (s.f.). Cooperación con Portugal. Recuperado de http://www. eucyl.jcyl.es/web/jcyl/Eucyl/es/Plantilla66y33/1277999714467/_/_/_ 
Kramsch, O. y Hooper, B. (2004). Cross-border governance in the European Union. Nueva York, Estados Unidos: Routledge.

Lois, R. C. y Carballo, A. (2015): La frontera hispano-lusa en la actualidad: Una visión geográfica. Revista de Historiografia, (23), 191-214.

López, L. (2005). La Raya Ibérica como espacio de atracción y cooperación. Economía de las Comunidades Autónomas: La Raya Ibérica: Centro-sur. Papeles de Economía Española, (21), 3-13.

Lucio, J. de y Barrios, S. (2002). Integración económica y efecto frontera en la Península Ibérica. Economía Internacional: Estudios Recientes, (796), 121-129.

Macorra, L. F. de la (2005). Caracterización económica de la Raya Ibérica. Economía de las Comunidades Autónomas: La Raya Ibérica: Centro-sur. Papeles de Economía Española, (21), 15-30.

Marks, G. y Hooghe, L. (2004). Contrasting visions of multi-level governance. En I. Bache y M. Flinders (Eds.), Multi-level governance (pp. 15-30). Oxford, Inglaterra: Oxford University Press.

Márquez, J. A. (2012). El desencuentro entre la frontera y La Raya: El fracaso de las politicas territoriales de desarrollo en la Eurorregión Andalucía-Algarve-Alentejo. Recuperado de http://www.acacr.es/discursos/E1\%20desencuentro\%20 entre $\% 201$ a $\% 20$ frontera $\% 20 y \% 201 \mathrm{a} \%$ 20raya.pdf

Martín, B. (2012). La Iniciativa Interreg en Alentejo-Algarve-Andalucía: De la conectividad básica a las estrategias globales de desarrollo del territorio. Cuadernos de Cooperación Territorial Europea, (2), 38-41.

Medeiros, E. J. (2010). Old vs recent cross-border cooperation: Portugal-Spain and Norway-Sweden. Area, 42(4), 434-443.

Medeiros, E. J. (2015). Territorial impact assessment and cross-border cooperation. Journal Regional Studies, 2(1), 97-115.

Medina, E. (2007). Perfiles estructurales de la frontera hispano lusa (la Raya). En E. Figueira, M. Saudade y M. M. Serrano (Coords.), Questões Sociais Contemporâneas (pp. 137-150). Centro de Investigação em Sociologia e Antropologia "Augusto da Silva”. Évora, Portugal: Universidade de Évora.

Ministerio de Hacienda y Administraciones Públicas del Reino de España (MINHAP). (2014). La cooperación transfronteriza realizada por las entidades territoriales españolas. Secretaría de Estado de Cooperación Territorial. Dirección General de Cooperación Autonómica. Recuperado de http://www.seat.mpr.gob.es/dam/es/ portal/areas/politica_autonomica/participacion-ccaa-eu/Menu_Coop_TransfrCooperacion/Coop_Transfronteriza/parrafo/00/text_es_files/2013_04_Informe-_web_cooperacion_transfronterizax.pdf

Minondo, A. (2007). The disappearance of the border barrier in some European Union countries' bilateral trade. Applied Economics, 39(1), 119-124.

Morata, F., y Noferini, A. (2014). Gobernanza y capacidades institucionales en la frontera pirenaica (Documento de trabajo núm. 326). Barcelona, España: Institut de Ciències Polítiques i Socials, Universitat Autònoma de Barcelona.

Nitsch, V. (2000). National borders and international trade: evidence from the European Union. The Canadian Journal of Economic/Revue Canadienne d'Economique, 33(4), 1091-1105. 
North, D. (1990). A transaction cost theory of politics. Journal of Theoretical Politics, Cambridge University Press, 2(4), 355-367.

Oliveras, X.; Durà, A. y Perkmann, M. (2010). Las regiones transfronterizas: nuevas evidencias de la regionalitzación de la cooperación transfronteriza en Europa (1958-2007). Documents d'Anàlisi Geogràfica, 56(1), 21-39.

Olson, M. (1965). The logic of collective action: Public goods and the theory of groups. Massachusetts, Estados Unidos: Harvard University Press.

Perkmann, M. (2003). Cross border regions in Europe. Significance and drivers of regional cross-border cooperation. European Urban and Regional Studies, 10(2), 153-171.

Perkmann, M. y Sum, N. L. (2002). Globalization, regionalization and cross-border regions. Basingstoke, Reino Unido: Palgrave Macmillan.

Pires, I. y Teixeira, J. A. (2002). A integraçao do mercado iberico: Novos ventos que casamento? En C. Cavaco (Coord.), Repensar Portugal na Europa, perspectivasde um país periferico (pp. 301-331). Lisboa, Portugal: Universidade de Lisboa.

Pires, I. y Teixeira, J. A. (Abril de 2003). Portuguese border regions: Will they be able to adapt to a global economy? Documento presentado en la Conferencia da RSA Reiventing Regions in the Global Economy, Pisa, Italia.

Podadera, P. y Calderón, F. J. (2014). La frontera hispano-lusa en el contexto de la integración europea: ¿Un futuro imperfecto? RUE: Revista Universitaria Europea, (20), 107-136.

Ponte, M. T. (2001). La Comunidad de Trabajo como instrumento dinamizador de la integracion. Tempo Exterior, (3).

Ponte, M. T. y Pueyo, J. (2006). La cooperación Galicia-Norte de Portugal en el nuevo entorno jurídico del Tratado Hispano-Portugués sobre cooperación transfronteriza entre entidades e instancias territoriales y de los instrumentos de cooperación interterritorial de la Unión Europea. Dereito: Revista Xurídica da Universidade de Santiago de Compostela, 15(1), 127-169.

Rhi-Sausi, J. L. y Conato, D. (2009). Cooperación transfronteriza e Integración en America Latina. Roma, Italia: IILA-cespI.

Rodrik, D. (2012). Una economía, muchas recetas: La globalización, las instituciones y el crecimiento económico. México Distrito Federal: Fondo de Cultura Económica.

Rosamond, B. (2010). New theories of European integration. En M. Cini y N. Pérez-Solórzano (Eds.), European Union Politics (pp. 104-122). Oxford, Inglaterra: Oxford University Press.

Rosell, C. (2012). Eurorregión Alentejo-Algarve-Andalucía: Cooperación transfronteriza en el horizonte 2020. Cuadernos de Cooperación Territorial Europea. (2), 23-31.

Scott, J. y Collins, K. (1997). Inducing transboundary regionalism in asymmetric situations: The case of the German-Polish border. Journal of Borderland Studies, 12(1-2), 97-121.

Taylor, P. (1994). The state as container: Territoriality in the modern world-system. Progress in Human Geography, 18(2), 151-162.

Wackermann, G. (2003) Les frontières dans un monde en mouvement. París, Francia: Ellipses Collection Carrefours. 


\section{Material legislativo}

Additional Protocol to the European Outline Convention on Transfrontier Co-operation between Territorial Communities or Authorities. Noviembre 9 de 1995. European Treaty Series n. ${ }^{\circ} 159$.

Convenio-Marco Europeo sobre cooperación transfronteriza entre comunidades o autoridades territoriales hecho en Madrid. Mayo 21 de 1980.

Convenio-Marco Europeo sobre cooperación transfronteriza entre comunidades o autoridades territoriales hecho en Madrid, de 21 de mayo de 1980. Artículo 2.1. Boletín Oficial del Estado [вов] n. ${ }^{\circ}$ 248. Octubre 16 de 1990. Recuperado de https://www.boe.es/boe/dias/1990/10/16/pdfs/A30270-30279.pdf

Convenio-Marco Europeo sobre cooperación transfronteriza entre comunidades o autoridades territoriales hecho en Madrid, de 21 de mayo de 1980. Artículo 2.2. Boletín Oficial del Estado [воE] n. ${ }^{\circ}$ 248. Octubre 16 de 1990. Recuperado de https://www.boe.es/boe/dias/1990/10/16/pdfs/A30270-30279.pdf

Protocol No. 2 to the European Outline Convention on Transfrontier Co-operation Between Territorial Communities or Authorities Concerning Interterritorial Co-operation, mayo 5 de 1998. Europe Treaty Series n. ${ }^{\circ} 169$.

Protocol No. 2 to the European Outline Convention on Transfrontier Co-operation Between Territorial Communities or Authorities Concerning Interterritorial Co-operation, Artículo 1. Mayo 5 de 1998. European Treaty Series n. ${ }^{\circ} 169$. Recuperado de https://www.coe.int/en/web/conventions/full-list/-/conventions/ $\mathrm{rms} / 090000168007 \mathrm{f} 2 \mathrm{cb}$

Protocol No. 3 to the European Outline Convention on Transfrontier Cooperation Between Territorial Communities or Authorities Concerning Euroregional Co-operation Groupings (ECGs). Noviembre 16 de 2009. European Treaty Series n. ${ }^{\circ} 206$.

Reglamento (CE) n. ${ }^{\circ}$ 1082/2006 del Parlamento Europeo y del Consejo, sobre la Agrupación Europea de Cooperación Territorial (AECT). Julio 5 de 2006. Diario Oficial de la Unión Europea.

Reglamento (UE) n. ${ }^{\circ}$ 1302/2013 del Parlamento Europeo y del Consejo, por el que se modifica el Reglamento (CE) n. ${ }^{\circ}$ 1082/2006 sobre la Agrupación Europea de Cooperación Territorial (AECT) en lo que se refiere a la clarificación, a la simplificación y a la mejora de la creación y el funcionamiento de tales agrupaciones. Diciembre 17 de 2013. Diario Oficial de la Unión Europea.

Tratado de Shengen. (1991).

Tratado de Valencia. (2004). Tratado entre el Reino de España y la República Portuguesa sobre cooperación transfronteriza entre entidades e instancias territoriales, hecho en Valencia. Octubre 3 de 2002.

Versión Consolidada del Tratado Constitutivo de la Comunidad Europea. Diciembre 24 de 2002. Artículo 2. Diario Oficial de las Comunidades Europeas. Recuperado de https://www. boe.es/legislacion/enlaces/documentos/ue/Trat_EC_consol.pdf 
Francisco José Calderón Vázquez

Español. Doctor en Ciencias Económicas (Universidad de Málaga). Licenciado en Derecho (Universidad de Granada), Profesor del Departamento de Economía Aplicada, Facultad de Ciencias Económicas y Empresariales. Líneas de Investigación: economía regional (fronteras, unión europea), desarrollo económico, turismo, globalización (cooperación internacional, migraciones, relaciones internacionales). Publicaciones recientes: (2016) Repensando la frontera africana de Castilla: ¿Una frontera interétnica? Frontera Norte, 28(55); (2015) Repasando la frontera hispano-portuguesa: Conflicto, interacción y cooperación transfronteriza. Estudios Fronterizos, 16(31), 65-89. 